\title{
Semantic Form as Interface
}

\section{Preliminaries}

The term interface had a remarkable career over the past several decades, motivated largely by its use in computer science. Although the concept of a "surface common to two areas" (Oxford Advanced Learner's Dictionary, 1980) is intuitively clear enough, the range of its application is not very sharp and well defined, a "common surface" is open to a wide range of interpretations. As an initial guideline for the following considerations, the definition in (1) might be useful:

(1) Interface: a point at which independent systems or diverse groups interact; computer science: The point of interaction or communication between a computer and any other entity, such as a printer or human operator.

(The American Heritage Dictionary, 1992)

It should be clear enough that the notion of interface does not only have a strong metaphorical flavor, but that its application is therefore in most cases just a heuristic move. With this proviso in mind, it might be useful to explore some possibilities of its application with respect to a specific domain. To this effect, I will consider an interface as a formal means, by which two separate systems interact. Hence I will not use it for the link between two domains or parts of the same system. I will not talk e.g. of the interface between words and sentences or between sentence and text, just as we would not talk about the interface between a book and its pages. But it might be appropriate to assume an interface between e.g. articulation and perception or between language and space. More explicitly, I will assume that two systems $A$ and $B$ have an interface with the structure $C$, if the following holds:

(2) (a) A and B are structurally different in relevant respects.

(b) $A$ and $B$ share a system $C$, which represents a restricted isomorphism between partial structures and processes of $A$ and $B$.

(c) $A$ interacts with $B$ in terms of $C$, i.e. there are systematic effects of $A$ on $B$ (and vice versa), which are mediated through $\mathrm{C}$.

This is still a rather provisional characterization, but it should be sufficient for the present purpose. For the sake of illustration, think of a pocket calculator and the obvious, but by no means trivial prerequisites on the basis of which it is to be operated. Evidently, the internal organization of the pocket calculator differs essentially from that of its putative user. But they share a subsystem by means of which they systematically interact. This system consists of the calculator's keys and display, both using the digits 0 to 9 with decimal coding. Further keys indicate arithmetical operations, the result of which is represented by the display. It is to be noted, by the way, that in this case the interface, by which A influences $B$ is not identical to that by which system $B$ returns the effects to $A$. In other words, the interface between two systems is not necessarily symmetrical. 
Notice furthermore that, in line with (2a), the interface structure will usually be related to rather different operations and structures within each of the two interacting systems. This is not at variance with a necessary partial equivalence, on which the interface is based. Without partial equivalence, systematic interaction between two systems would in fact be impossible. Thus the pocket calculator clearly realizes processes that are quite different from those of the human user of the machine, even though both are equivalent to the extent to which they are bound to and controlled by principles of arithmetic and decimal coding of numbers.

In what follows, I will suggest that the heuristic use of the notion interface may shed some light on problems that are traditionally dealt with in terms different levels of representation, such as Conceptual Structure and Logical or Semantic Form.

\section{The Conceptual Framework}

According to uncontroversial assumptions, a natural language relates the form of its expressions to their meaning, and vice versa. Hence a natural language is a system of knowledge that creates a systematic correspondence between the elements of two rather different domains, viz. the form of linguistic expressions and their meanings. The structure of these two domains is based on corresponding systems of mental organization, that I will abbreviate, following Chomsky (1995), as A/P und C/I, respectively, where $A / P$ is the complex system controlling articulation and perception, i.e. the production and recognition of signals, and $\mathrm{C} / \mathrm{l}$ abbreviates the even more complex systems of conceptual and intentional representations, in terms of which the human organism deals with its external and internal environment. Schematically, this can be represented as follows:

\section{(3) Signal $<====>\mathrm{A} / \mathrm{P} \quad<===$ Language $===>\mathrm{C} / \mathrm{l} \quad<====>$ Environment}

In view of this schema, one might be tempted to apply the interface-metaphor to the system of language as a whole, observing that its task is to connect the systems of $\mathrm{A} / \mathrm{P}$ and $\mathrm{C} / \mathrm{l}$. This move would be heavily misleading, though, for at least two reasons. First, the basic principles of A/P must be essentially different from those of $\mathrm{C} / \mathrm{I}$, because elements of $A / P$ are basically linear patterns of linguistic signals, while the structures of $\mathrm{C} / \mathrm{l}$ are of a radically different type, capable to integrate conditions of all aspects of conceptually organized experience, as will be discussed in somewhat more detail below. Hence no reasonable subset of common elements provides a system of shared representations. Second, this heteromorphy of $A / P$ and $C / I$ is not an incidental deficit, but rather the fundamental condition for the unprecedented nature of the human language capacity, namely the emergence of a recursive mapping between two completely heterogeneous domains. The crucial point is that natural languages are combinatorial systems of symbolic signs, i.e. signs with a necessarily conventional relation between form and meaning and inherent recursive compositionality.

In other words, the fundamental difference between $\mathrm{A} / \mathrm{P}$ and $\mathrm{C} / \mathrm{I}$ does not only prevent an interface between form and meaning, but it is for the same reason the source of the unique nature of the language faculty and its special status among the mental capacities. 
Hence a natural language must be construed as a mental system, that accounts for the relationship indicated in (3) in two respects, that must be clearly distinguished. First, it must accommodate the incommensurable structures of two different systems $\mathrm{A} / \mathrm{P}$ and $\mathrm{C} / \mathrm{I}$, and second, it must compute a bridge between the accommodated structures. These different requirements are represented in (4), following the Minimalist Program of Chomsky (1995), which construes the internal or I-Language as a system that generates pairs of representations belonging to the Phonetic Form PF and the Logical Form LF, respectively, assuming that the correspondence between $A / P$ and $C / I$ is mediated through these linguistic levels of representation:

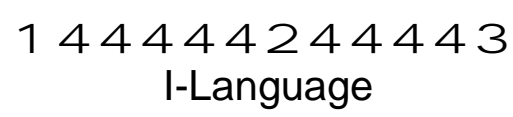

The present paper will focus on the first of these requirements, i.e. on linguistic representations that are connected to extra-linguistic structures. Even though this connection is clearly determined by the respective extra-linguistic systems, it still depends crucially on intra-linguistic conditions, notably those on the sub-systems of Phonetic and Logical Form, to which the interface-metaphor most naturally applies.

The conditions imposed by the system of I-Language on the Interface Representations include in particular the Principle of Representational Economy, which precludes the appearance of vacuous elements. With respect to the interface levels, Representational Economy amounts to the requirement (5):

(5) Full Interpretation: LF and PF must not contain elements that are not interpretable in $\mathrm{C} / \mathrm{I}$ and $\mathrm{A} / \mathrm{P}$, respectively.

The rationale of this principle is the assumption that there are elements or features of I-Language that have no extra-linguistic realization, but merely govern the computational processes that relate LF and PF. The details of these operations will not concern us here. As a consequence of the principle of Full Interpretation, neither PF nor LF allow for elements that are vacuous with respect to the external systems they interact with. Thus PF cannot contain e.g. degrees of stress on obstruents, as only syllables can participate in stress patterns. Conversely, though, interpretations of $\mathrm{PF}$ in A/P may exhibit systematic as well as accidental distinctions that cannot be reflected in PF. A simple case in point are the properties of voices of male as opposed to female speakers, or the different mood accompanying an utterance of Good morning! that the listener might well identity. This suggests that the relation between A/P and PF is a homomorphism in so far as PF abstracts away from A/P in systematic respects. Although this seems to be correct in crucial respects, it is not the whole story, though, as we will see shortly.

Before turning to the main topic of this paper, viz. the interaction of I-Language with $\mathrm{C} / \mathrm{l}$, a terminological adjustment is to be made. Within the Minimalist Program, the interface of I-Language with $\mathrm{C} / \mathrm{I}$ is taken to be LF, which is a syntactic level, made up from elements and categories to be chosen from the store of lexical items. Now, while at PF lexical items are considered as complex structures, consisting of features, segments, and syllables, at LF they are treated as basic elements - verbs, nouns, prepositions, etc. -, essentially without internal structure, although from a 
different perspective they are nothing but collections of features, including semantic ones. As the internal structure of lexical items and its systematic consequences will turn out to be essential for the conceptual interpretation of I-Language, I will assume it to be represented by the Semantic Form SF, a representational level that actually provides the interaction of I-Language with the conceptual and intentional conditions of language use. More specifically, I will consider lexical items like long, short, in, under, open, etc. not just as sets of features with no semantically relevant internal structure, but as systematic configurations, which are relevant for I-Language as well as its interpretation in C/I. Hence the schema (4) should be changed to (6):

$$
\mathrm{A} / \mathrm{P}<====>\mathrm{PF} \longleftrightarrow \mathrm{SF}<====>\mathrm{C} / \mathrm{l}
$$

This modification raises the question of how SF relates to syntax in general and LF in particular. Without going into the details, I will assume, however, that SF reduces to LF, if the distinction between lexical items and the SF-primes of which they are made up is ignored ${ }^{1}$. To put it the other way round, SF emerges from LF, if the internal organization of lexical items with respect to their semantic interpretation is made explicit - just as PF "spells out" the internal structure of lexical items with respect to their phonetic interpretation. These remarks leave untouched a large number of questions, which are essential for the computational mapping between PF and SF. But the basic condition concerning the SF-C/I-Interface should be clear enough: The conceptual interpretation of linguistic expressions has to take into account the internal structure of words like tall as opposed to short- just as the articulatory interpretation has to deal with the structure of tall as opposed to call.

\section{Some Empirical Observations}

Besides obvious differences, there are important similarities between the two interfaces of I-Language. It might therefore be useful to have a look at the interpretation of $\mathrm{PF}$, before the more controversial issues concerning interpretation of SF are taken up.

It is a well known observation, that $P F$ is generally more restrictive than $A / P$ : It abstracts away from various types of information that are present in the signal and play a systematic role in speech processing. Properties distinguishing male and female voices, characteristics of personal identity, age or mood of the speaker are among the obvious factors that appear in $\mathrm{A} / \mathrm{P}$ and are recognized by speaker and hearer alike, but do not enter PF. This suggests that PF is a homomorphism with respect to the patterns belonging to $A / P$. The interaction of I-Language and $A / P$ would thus reduce the information under perception, while in speech production $A / P$ adds amendments to the information of PF. This is not the whole story, though. It is easy to imagine conditions of impoverished or defective acoustic conditions that nevertheless lead to complete, normal representations at PF. More interestingly, PF may systematically reflect distinctions that are not always realized in A/P. A case in point are stress patterns, whose systematic distinctions in PF often go beyond usual

\footnotetext{
${ }^{1}$ Taking the structure of SF, to which we turn below, as input for the mapping from SF to PF, we can conversely construe LF as the result of adding the morpho-syntactic categorization on the basis of the lexical items from which the SF-representation is made up
} 
phonetic realizability, nevertheless becoming relevant, e.g. under metrical conditions of poetry.

In short, PF reconciles conditions of I-Language and articulation/perception. Its representations are made up from basic elements, which have a systematic status in both domains and are combined by principles that both domains allow for. Each of these domains may involve specifications, which the other ignores, but the shared conditions nevertheless guarantee automatic and reliable matching. ${ }^{2}$

Turning now to the semantic interface, we notice first a well known and extensively documented phenomenon, viz. the richness of conceptual distinctions compared to their rather sparse reflection in linguistic representations. Like PF, although in rather different respects, SF abstracts away from specifications provided by the conceptual interpretation. The point might be illustrated by two simple examples:

(7) I finished the book a while ago.

Depending on whether the speaker is the author or a reader of the book, whether the book is a novel or a research report, whether it comes from a public library or is private property, quite different situations can be referred to. Similarly for an appropriate utterance of (8), Paul may be disappointed by the new building he was shown around, or he may dislike the atmosphere among the colleagues he was working with or the job he had as an employee of the institute. Several other interpretations might come to mind, which are conceptually, but not linguistically distinct.

(8) Paul didn't like the institute.

Notice that the different interpretations of cases like (7) or (8) do not turn on ambiguity of any sort, neither structural like (9a) nor lexical like (9b).

(9)(a) He had secret plans to leave.

(b) The post was unreliable.

Thus while the conceptually different interpretation of cases like (9) is systematically reflected in SF, either by different choice or by different combination of elements, no such account is assumed for (7) or (8), which instantiate one type of what is usually called underspecification. It is easy to see that this sort of lacking specificity is an ubiquitous phenomenon of natural language. ${ }^{3}$

What is less well established is the fact that SF and its foundation in I-Language may support distinctions and relations that are not reflected in $\mathrm{C} / \mathrm{l}$, in other words, SF can also be more specific than its interpretation in $\mathrm{C} / \mathrm{l}$. Cases of this sort become visible

\footnotetext{
${ }^{2}$ This observation is only apparently at variance with the principle of Full Interpretation indicated in (6). The point is not that PF may contain elements that are not interpretable in $A / P$, but rather that not all relations and distinctions among interpretable elements of $P F$ are reflected in $A / P$.

${ }^{3}$ Not only everyday utterances with obvious conceptual context rely on this sort of unspecificity. An important part of poetry is based on just this phenomenon, as is obvious from e.g. Hölderlin's verses:

Die Linien des Lebens sind verschieden

Wie Berge sind und wie der Länder Grenzen.
} 
in case of differences projected on the same situation by different languages. An obvious case in point is (10), an inscription presented at the Berlin subway entrance:

$$
\text { Please validate your ticket Bitte das Ticket entwerten }
$$

Every dictionary correctly characterizes entwerten (devaluate) and validate as antonyms, or rather as designating inverse processes, but the two expressions in (10) are nevertheless understood as requesting the same activity. Thus different aspects of the same state of affairs are (conventionally) fore-grounded in English and German.

A different, and more complicated pattern of distinctions can be seen in (11):

(11) (a) Peter took off his hat

(b) Peter took off his pullover

(c) Peter undressed (completely) (a') Peter nahm/setzte den Hut ab

(b') Peter zog den Pullover aus

(c') Peter zog sich (ganz) aus

In German, two types of activity, which English lumps together in (a) and (b), are distinguished on the basis of different objects in ( $\left.a^{\prime}\right)$ and ( $\left.b^{\prime}\right)$. On the other hand, getting rid of specified objects and of the clothes altogether, as in ( $\left.b^{\prime}\right)$ and ( $\left.c^{\prime}\right)$, respectively, are covered by the same verb in German, while they are clearly distinguished by (b) and (c) in English. Actually, things are far more complex than the examples in (11) indicate - not only because ausziehen is multi-ways ambiguous with respect to readings not relevant here and in ways that differ essentially from comparable ambiguities of take off, as every dictionary shows, but also because the contrast between etwas ausziehen in ( $\left.b^{\prime}\right)$ and sich ausziehen in ( $c^{\prime}$ ) is not just an instance of underspecification, but rather a special type of ambiguity. ${ }^{4}$

Another type of semantic distinctions that does not represent conceptual differences turns on locative relations of the following sort:
(12)(a) the cup on the table
(a') die Tasse auf dem Tisch
(b) the handle on the door
(b') der Griff an der Tür

While direct contact between an object and a supporting surface is realized by the same relation in English, in German requires a distinction to be made between the relation to a horizontal surface and the relation to vertical surface. Again, further complications show up, other relations and additional conditions are taken into account. Bowerman (1996) discusses a wide range of distinctions made in different languages with respect to spatial relations.

Semantic distinctions like those in (10), (11), or (12) turn on the content of individual lexical items, not on general principles. There might be more general conditions, however, which associate the same state of affairs with systematically different

\footnotetext{
${ }^{4}$ The two readings that need to be lexically distinguished can be made visible by the ambiguity of (i), where ihn can be replaced by den Mantel (his coat), as in (ii), or seinen Sohn (his son), as in (iii), with clearly different relations in SF.

$\begin{array}{lll}\text { (i) Peter zog inn aus } & \text { (ii) Peter zog den Mantel aus (iii) Peter zog seinen Sohn aus }\end{array}$

Both relations are realized by grammatically different arguments in (iv):

(iv) Peter zog seinem Sohn den Mantel aus. (Peter took his son the coat off)

It might be noted that these intricacies are not merely a lexical anomaly of the verb ausziehen - they are mirrored by parallel phenomena that appear with the verb anziehen.
} 
semantic representations in different languages. For instance, Levinson (1996) describes three types of spatial orientation by which different languages identify the front/back- and left/right-direction of an object. In systems with intrinsic orientation the direction depends on the object, in systems of relative orientation, it depends on the observer, and in systems of absolute orientation, it depends on the overall environment. Levinson's observations are, once again, more intricate than this rough summary indicates ${ }^{5}$, but the main point should be clear enough: There are alternative ways, according to which different languages assign spatial structure to the same situation.

Notice, however, that more or less systematic distinctions in SF, which don't have different interpretations in $\mathrm{C} / \mathrm{l}$, are not at all in conflict with principle (5) of Full Interpretation. (5) prevents conceptually vacuous elements or relations in SF, but it doesn't require distinct interpretations for different elements. ${ }^{6}$ And the fact that, conversely, SF ignores a great number of differences present in $\mathrm{C} / \mathrm{l}$, as indicated in (7) and (8), is even less in conflict with Full Interpretation. As a matter of fact, if a conceptual distinction does appear in $\mathrm{C} / \mathrm{I}$, then it can in principle be represented also in SF.

In sum, the interaction between conceptually represented experience and the linguistic representations that most directly correspond to it is open to interpolation in various ways. But in spite of this flexibility, there must be a reliable common core $\mathrm{C}$ in the sense noted in (2) above, by means of which the interaction succeeds. The following sections will be concerned with minimal assumptions to be made in this sense with respect to the interface between $\mathrm{C} / \mathrm{l}$ and linguistic knowledge.

\section{Minimal Conditions on SF}

Interfaces like SF and PF are subject to two types of requirements: First, they must share to some extent the format of the system they interact with. Second, they must be composed of basic elements that are motivated by and can be interpreted in the interfacing systems. In case of PF, the representational format derives from the time course determining the articulation and perception of the signal. More specifically, the skeleton of PF is a sequence of time slots (13), where each $X$ is a slot to which the PF-elements are linked:

\footnotetext{
${ }^{5}$ It is, in particular, not obvious, to what extent different linguistic representations of the same spatial scenario induce conceptual differences that go beyond their verbalization, including inferences about spatial relations. But this is a separate issue that need not concern us here.

${ }^{6}$ There are, of course, numerous ways to map different elements onto identical realizations. To give an analogy from music, enharmonic keys like Cis and Des major have a well defined, different position in the tonal system, although their acoustic realization is indistinguishable. See also fn 2 with respect to PF.

${ }^{7}$ In other words, different thoughts can in principle be expressed by different words. More precisely: for every conceptual distinction $\mathrm{C} / \mathrm{l}$ provides for, there is in any natural language $\mathrm{L}$, a (possibly complex) expression whose SF explicitly represents that distinction. This is, by the way, the principle defended in Searle (1969) under the term expressibility as a defining property of natural language. It must be added, though, that this principle is confined to conceptual distinctions - whatever that means. It does not apply to representations that are inherently restricted to analogical representation. Thus, one can distinguish two persons by name, but one cannot verbally express the difference of their faces. These are intricate problems, which cannot appropriately be pursuit here.
} 
The linear ordering inherent in this sequence provides also the combinatorial principle underlying the PF-representations. The basic elements in terms of which PF is organized are features (or feature values), which are systematically linked to the skeletal positions, such that formally three-dimensional representations emerge, organized along the skeletal core. The feature values, functioning as primes within $\mathrm{PF}$, represent properties, which in A/P impose conditions on signal production and perception, i.e. on place, manner, and agent of articulation. Although this sketch is oversimplified in various respects ${ }^{8}$, the way in which $P F$ is related to A/P should be clear at least in principle: The sequential organization of PF as well as the "content" of its primes is due to $A / P$, the relations within $P F$ are determined by the rules and principles of I-Language.

The situation is comparable for SF, although remarkably more complex in at least two respects. First, there is no evident base line which determines a straight and obvious representational format. There is nothing comparable to the time course of the signal (which, by the way, controls spoken as well as signed languages, and holds even for the derivative format of written language). No direct and obvious principle of this type organizes the totality of situations or states of affairs which linguistic expressions are about. ${ }^{9}$ Hence no natural condition on the dimensionality of SF seems to be given in advance. Second, the variety of domains in which primitive elements of SF are to be interpreted exceeds by far those of $A / P$, as is easily to be recognized: $A / P$ has to recruit motor control of signal-production and perceptual analysis of the signals thus produced, while $\mathrm{C} / \mathrm{I}$ must integrate all aspects that are involved in the organization and control of behavior. I will return to the second problem in section 5 and 6 , turning first to the representational format of SF.

The general nature of $C / I$ can, unlike that of $A / P$, neither emerge from the temporal structure of expressions, nor from spatial or any other basic dimensionality, it must rather encompass conceptual configurations of any kind, including a-temporal and non-spatial structures. Hence a sufficiently abstract combinatorial framework must be assumed to support and control SF. A general principle that meets this condition is the functor-argument-combination, according to which a functor $X$ applies to an argument $Y$, with no linear ordering between $X$ and $Y$. This principle implies that elements of SF be assigned to types which specify their combinatorial properties. The relevant type system and the pertinent assignment can be defined as follows:

(14) (a) If $\alpha$ and $\beta$ are types, then $\langle\alpha, \beta\rangle$ is a type.

(b) If $\mathrm{X}$ is of type $\langle\alpha, \beta\rangle$ and $\mathrm{Y}$ is of type $\alpha$, then $[\mathrm{X} \mathrm{Y}]$ is of type $\beta$.

\footnotetext{
${ }^{8}$ For a more detailed exposition, see e.g. Halle $(1983,1995)$. - It should be added, that although A/P is to be construed as normally dealing with spoken language, it must alternatively include the basis for sign language, i.e. visual perception of gestural articulation. See Bierwisch (2001) for some discussion of pertinent consequences.

${ }^{9}$ It must be stressed, that the temporal nature of mental operations, including the production or comprehension of utterances is an undeniable fact, which must not be confused, however, with the completely independent (a)temporal nature of their meaning. An utterance like (i) is, of course, formulated and interpreted in real time, but that does not imply that John preceded the woods or the period of three hours in any sense.

(i) John walked through the woods for three hours

See Bierwisch and Schreuder (1992) for some discussion of these matters.
} 
This seems to be the minimal framework which the organization of SF could rely on in view of the indefinite or open dimensionality of $\mathrm{C} / \mathrm{I}^{10}$.

The next point to be noted is the fact that a functor $\mathrm{X}$ may have properties according to which it must apply to two (or more) arguments. The characterization of those types is made possible by the recursive nature of definition (14a), which allows both $\alpha$ and $\beta$ to be complex types of the sort defined in (14)(a): If $\alpha$ and $\langle\alpha, \beta\rangle$ are types, then $\langle\alpha,\langle\alpha, \beta\rangle\rangle$ is again a type, viz. the type of a functor that combines with two elements of type $\alpha$ to get a complex element of type $\beta$. As a matter of fact, (14) allows for combinations of unlimited complexity ${ }^{11}$. It is an empirical question, to what extent these possibilities are made use of, and what boundaries might actually constrain their exploitation.

The most important point in this respect is the constraint already embodied in (14), viz. the condition that functor types can only be binary, combining a functor with just one argument. A functor with more than one argument, actually a ubiquitous reality, must therefore belong to a type of the sort (15), indicating that a many-placed functor combines with its arguments in hierarchical steps.

$$
\left\langle\alpha_{1},\left\langle\alpha_{2},\left\langle\ldots\left\langle\alpha_{n}, \beta\right\rangle\right\rangle\right\rangle\right\rangle .
$$

This condition corresponds directly to the fact that no sequential ordering is available to represent structural relations in SF. Thus in order to keep apart the different relations a functor has to its different arguments, hierarchical distinctions must be made. If for instance (16)(a) and (b) are assumed to be different according to standard notational conventions, according to which a functor $\mathrm{R}$ is placed between its first and second argument, then (17)(a) and (b) would be the corresponding hierarchical representation, where hierarchy is indicated by bracketing, while linear order is irrelevant. ${ }^{12}$
(16)(a) a R b
(b) $\mathrm{b} R \mathrm{a}$
(17)(a) $[a[R b]]$
(b) $\left.\left[\begin{array}{ll}\mathrm{a} & \mathrm{R}\end{array}\right] \mathrm{b}\right]$

\footnotetext{
${ }^{10}$ One might object that an even more elementary framework is the system of Bare Phrase Structure proposed in Chomsky (1995), according to which two elements $X$ and $Y$ are combined into $[X Y]$ by the operation Merge, which assigns [X Y] simply the categorization (i.e. the "label") of its head, say X. Hence no functor types of the sort defined in (14a) must be assumed. Notice, however, that the condition by which the head $X$ selects its complement $Y$ must now be given in some other way. Therefore the framework as a whole cannot be simpler or more general than the one proposed in (14). ${ }^{11}$ The proposal to characterize the combinatorial structure of natural languages by recursive type systems of the sort adopted here has been criticized e.g. in Schnelle (1999) as making the claim that knowledge of language is based on explicit mastery of fully fledged modern logic and type structure. However, by the same token, one would have to reject the claim that knowledge of natural numbers is appropriately characterized by the recursive successor operation, arguing that elementary calculation must not imply explicit recourse to e.g. Peano's axioms. In other words, the power of a theoretical system is not to be confused with its use to under restrictive boundary conditions.

${ }^{12}$ Hence (17a) could just as well be written as [ [ R b ] a ]. - To avoid notational confusion, I would emphasize, that according to (14), the type $\langle\alpha, \beta\rangle$ is strictly different from $\langle\beta, \alpha\rangle$. What is important is the fact, that the type system imposes no ordering on the elements combined, the claim is not, that the notation of types has no ordering. This consequence could be avoided, if we would write e.g. $\langle\alpha\langle\beta\rangle\rangle$ instead of $\langle\alpha, \beta\rangle$, which could then be construed as equivalent to $\langle\langle\beta\rangle \alpha\rangle$. Notations like these would deviate, however, from standard notational conventions for type systems, which I have adopted here.
} 
Strictly speaking, in SF linear order does simply not exist. As already noted, the time dependence of actual processes of the formation of thoughts and inferences must not be misconstrued as a structural property of SF - or C/l, for that matter. ${ }^{13}$

Less clear constraints delimit the set of types accessible to SF. This concerns first the inventory of basic types, from which functor types are derived by (14a), and second the finite subset of functor types that are actually needed for proper semantic representations. As to the first issue, it is a fundamental (and ultimately empirical) question, which basic types must be assumed for natural languages. I will adopt here the standard view that at least two basic types $e$ and $t$ must be distinguished, with $e$ as the type of entities, i.e. objects, substances, and events, and $t$ as the type of states of affairs or situations ${ }^{14}$. Further basic types might be imagined, but I will leave it at that. It should be clear, however, that basic types must not be confused with primitive elements. Semantic configurations assigned to $e$ or $t$ can in fact be of arbitrary complexity, while on the other hand functors of complex types may be primitive elements of SF. Functor types based on $e$ and $t$ include n-place predicates (18a), n-place functions from individuals to individuals (18b), functions from propositions to propositions (18c), functions from predicates to predicates (18d), etc.:

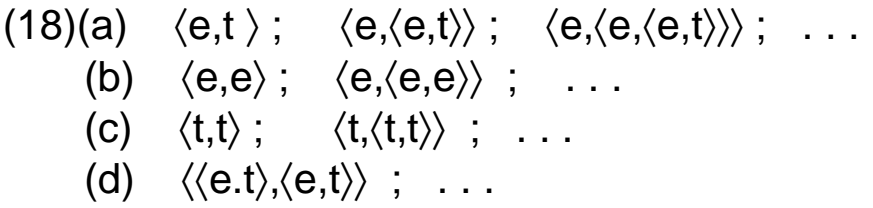

This open list directly leads to the question whether and how the set of functor types occurring in actual representations might be restricted, irrespective of the fact that (14) defines an infinite set of potential functors. While there is no point in setting up arbitrary limits for virtual structures, restricting e.g. the length of words or sentences or the complexity of concepts, there is an interesting limit deriving from the system's architecture. Remember, that the functor-argument-combination defined in (14b) necessarily reduces the complexity of the functor-type by applying the functor to its argument. Hence even in arbitrary complex configurations, types cannot become more complex than those of the basic functors the combination starts with. The range of types is thus automatically restricted by the set of primitive elements and their types ${ }^{15}$. If the set of primes is fixed and finite, an issue to which we will turn shortly, the range of types is obviously finite ${ }^{16}$. In any case, the type system determines a

\footnotetext{
${ }^{13}$ Consider e.g. a case like Who did he talk about, where the time course of production and comprehension is subject to different conditions, as the hearer normally assigns the Theme-role to who only when about is processed, while the speaker does it in advance, although the SF of the sentence is the same under both perspectives. See also fn.9. - Notice, incidentally, that no such asymmetry holds for PF, where linear ordering is after all a structural property.

${ }^{14}$ The considerations underlying this choice can be traced at least to Frege (1879) and have been pursued within the tradition of formal semantics, developed especially in Montague (1974), where the notational variant $t$ for truth-value bearer and $e$ for entity has been proposed. To adopt this convention is not an arbitrary choice, as the distinction seems to be fundamental. The general tenets of formal semantics, however, which sharply distinguish logical (objective) from mental (subjective) aspects of meaning, differ essentially from the present perspective, for which semantics is a matter of mental phenomena.

${ }^{15}$ Notice that this does not restricts the complexity of possible configurations in SF, since due to the recursive nature of the combination defined by (14b), functors as well as arguments can be arbitrarily complex, irrespective of the restricted complexity of their type.

${ }^{16}$ Two further constraints may be worth noticing: First, functors of type $\langle\alpha, \beta\rangle$ with basic $\alpha$ and $\beta$ define the indispensable minimum, since otherwise there would be no combination. Second, if definition (14b)
} 
restricted system that supports a potentially infinite set of representations with completely abstract dimensionality.

Notice that the type-system does not consist of elements of SF, just as the ordering of segments does not consist of elements of PF. The type system determines the format, not the content of representations of SF in much the same way in which formation and ordering of segments determines the format, but not the content of PF. In view of the interface-nature of SF, we are nevertheless faced with the question, how this representational framework corresponds to the structure of $\mathrm{C} / \mathrm{I}$ which SF interacts with, as the format of the interfacing systems must be compatible, as noted earlier. For obvious reasons, a simple and direct answer, comparable to the time course as basis for the ordering of segments in PF, is largely impossible. As a simple illustration, consider situations like (19), which could easily be extended by further variations:

(19)(a) he crossed it

(c) he knew it

(e) he prevented it

(g) he denied it (b) he followed him

(d) he forgot it

(f) he destroyed it

(h) he hated him

Whatever he, him, and it might represent, it is obvious, that the different dimensionality and conceptual nature of the situations, their spatial, temporal, causal, cognitive, emotional relations cannot be distinguished and reflected by the representational format, but only by the content of the elements from which the SF of the different verbs is made up. To put it the other way round: Combining elements in SF means nothing but the bare connection. Whatever shows up as a distinction in SF must either be a distinction in terms of combinatorial hierarchy, or must derive from the content of semantic elements entering this combination. These considerations were in fact the reason for adopting the abstract functor-argument-structure as the underlying format of SF. This leaves us with the final and in many respects central problem of the interface character of SF, viz. the nature of its basic elements and their interpretation.

It first seems necessary to distinguish between constants and variables. The nature of variables is an important and intricate problem within the tradition of logic. Without entering these discussions, I will just make the following assumptions, which should be uncontroversial:

(20) (a) SF provides an open set of variables $x_{1} x_{1}, x_{2}, \ldots$ for every type $\alpha$, in SF.

(b) Any two variables $x_{i}$ and $x_{k}$ are different - within and across types.

(c) Every variable $x$ allows for an unlimited number of or tokens, i.e. $x$ can have more than one occurrence within the same SF-representation.

of functional application - abbreviated in (i) - is supplemented by functional composition (ii), in order to provide a more general mode of combination, then every $n+2$-place functor can in principle be built up from $n$ 2-place functors, as sketched in (iii) for a 3-place functor with arguments of type $\delta, \gamma$, and $\varepsilon$ :
(i) $\alpha \cdot\langle\alpha, \beta\rangle \Leftrightarrow \beta$
(ii) $\langle\gamma, \alpha\rangle \bullet\langle\alpha, \beta\rangle \Leftrightarrow\langle\gamma, \beta\rangle$
(iii) $\langle\delta,\langle\gamma, \alpha\rangle\rangle \bullet\langle\alpha,\langle\varepsilon, \beta\rangle\rangle \Leftrightarrow\langle\delta,\langle\gamma,\langle\varepsilon, \beta\rangle\rangle\rangle$

(Combination is indicated by ' $\bullet$ ', functors follow their argument for perspicuity.)

As a consequence, 2-place functors would be the upper bound for the complexity of types to which all configurations can be reduced. - For more systematic exposition of the technicalities, see e.g. Partee et al.(1990). Whether these formal matters are empirically relevant or not, must be left open here. 
(d) A variable $\mathrm{x}$ of type $\alpha$ can be substituted according to systematic principles by basic or complex elements $\varphi$ of type $\alpha$, including variables of type $\alpha$.

Substitution and binding of variables belong to the operations by which the SF of complex expressions is constructed from the SF of their constituent parts. As the present paper focuses on interpreting, rather than constructing SF-representations, the principles of variable binding and substitution will be left aside, noting only that occurrences of the same variable must be treated alike. This holds also for the way in which variables function as interface-elements with respect to $\mathrm{C} / \mathrm{I}$, a problem to be taken up below.

Turning now to the proper semantic primes, i.e. the basic constants of SF, a number of crucial questions is to be faced. Is there a fixed and finite, possibly universal repertoire of basic semantic elements? What would be the size of this repertoire? What would be the origin and the interpretation of its elements? While there is more or less agreement about these questions with respect to PF, this is by no means the case for SF. Thus the assumption of a fixed, universal repertoire as part of the language faculty is widely accepted with respect to $\mathrm{PF}^{17}$, but essentially doubted or practically denied with respect to SF, e.g. in Fodor (1981). A closer look at the problems involved urges one to distinguish primes of different kinds.

In fact, Katz and Fodor (1963) distinguished already between so-called semantic markers like (Human), (Male), (Adult) with an essentially systematic status, and distinguishers like [has never married] or [lowest academic degree]. ${ }^{18}$ This rather provisional distinction reappeared in various forms and with different claims associated with basic elements of different sorts. Thus to a large extent, Generative Semantics, as reported e.g. in Dowty (1979) concerned itself with the systematic properties of primitives like CAUSE, BECOME, HUMAN, some of which reappeared as functional heads and light verbs in various versions of LF, such as Hale and Keyser (1993) and later work. I will return to these matters in section 6.

To conclude this outline, two examples illustrate SF-representations as discussed so far. (21)(a) indicates the lexical SF of the transitive verb open, (21)(b) is that of the adjective red. The variables $\mathrm{x}, \mathrm{y}, \mathrm{z}$ mark positions to be filled in by constituents the lexical items combine with, while the variable $s$ in (21a) eventually refers to the situation that instantiates the condition specified by the verb: ${ }^{19}$

\footnotetext{
${ }^{17}$ In view of the revealing discoveries about sign language, I have argued in Bierwisch (2001) that the basis for primes of PF in the Language Faculty should presumably be assumed to determine only the organizational principles, instead of the specific content of the repertoire, such that the structural conditions can be realized by alternative modalities, relating e.g. to motor control of either the vocal tract or the hand.

${ }^{18}$ These are, of course, highly provisional examples for the purpose of illustration only. In later work, e.g. Katz (1972), the theoretical relevance of distinguishers was (inappropriately, I believe) very reduced, while semantic markers were considered as potentially complex, systematic configurations. The initial distinction was nevertheless a helpful proposal. It inspired necessary disputes that are not really settled yet.

${ }_{19}$ Technically, this role of variables is to be expressed by operators, which make the variables available for substitution by the SF of complements. Thus (i) sketches a more complete version of the entry for transitive open :
}

(i) / open / [ +V ] $\lambda y \lambda x \lambda s \quad\left[{ }_{t} S[\langle e, t\rangle\right.$ INST [t $x$ ACT ] [ $\langle t, t\rangle$ CAUSE [t BECOME [t OPEN $\left.\left.\left.\left.y]\right]\right]\right]\right]$ For details about the operators specifying argument positions see e.g. Bierwisch (1997). 
(21)(a)

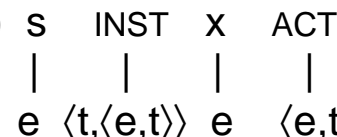

t

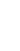

(2n)

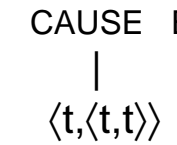

$\mid$

$\langle t, t\rangle$

$\langle e, t\rangle$

$\langle e, t\rangle \quad e$

$\mathrm{t}$

$t$ (b)

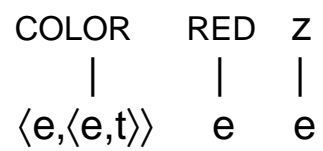

$\langle e, t\rangle$

t

$\langle e . t\rangle$

t

These examples also illustrate the general assumption that the SF of major lexical categories like Noun, Verb, Adjective are configurations of type t, specifying the more or less complex propositional condition that a lexical entry imposes on a situation it applies to. To combine these conditions - primarily by substitution and binding of variables - according to the syntactic structure of complex expressions is a matter of the computational system of I-Language. To relate the complex conditions thus specified, and their basic ingredients to the external and internal environment of the speaker/hearer is a matter of the $\mathrm{C} / \mathrm{I}$, to which we turn now.

\section{Basic Assumptions about $\mathrm{C} / \mathrm{I}$}

The systems of conceptual, intentional representation which SF interacts with are not a single, self-contained module of mental organization, but a highly complex aggregate of partially autonomous components with special biological foundation and different phylogenetic history. This aggregate includes the various perceptual capacities, the organization of motor control, of spatial and temporal orientation, the "Theory of Other Minds" supporting inter-individual coordination, and the effects of emotional evaluation, to give an incomplete and unsystematic list. It is clear that there must be essential modes of interaction among the various subsystems, such as visual, auditory and tactile perception controlling movement and action, or the various systems jointly supporting spatial orientation. The question, which systems are interrelated in which way, or which specific types of representation different subsystems rely on, cannot be pursued here in even remotely adequate way. We can only identify two types of problems that directly bear on the interface aspect of SF, indicating the differences and the resulting limits of the representational systems.

It must first be noted that most semantic theories simply do not acknowledge a representational difference between SF and C/I (or whatever would correspond to this distinction). Therefore the problem of characterizing representations of $\mathrm{C} / \mathrm{I}$ simply does not arise e.g. in standard versions of formal semantics. ${ }^{20}$ Assumptions about conceptual structure, if they are made explicit at all, are to a large extent plausible extrapolations from analyses dealing with SF (or Logical Form, for that matter).

\footnotetext{
${ }^{20}$ Notice that the distinction between extension and intension or Frege's Sinn and Bedeutung, familiar from formal semantics, must not be confused with the present distinction, even though their point is to deal with different aspects of meaning, albeit in a different respect.
} 
Exceptions to this claim can be found, however, in the domain of vision and spatial orientation. The vast literature on these matters warrants the claim that an essential distinction is to be made between spatial and semantic representations. ${ }^{21} \mathrm{~A}$ crucial point to be noted is the different format on which propositional and spatial systems (and may be others) are based, supporting different types of inferences and problem solving normally classified as thinking.

A concrete example which highlights the latter point is the notion of mental models discussed in Johnson-Laird (1994). A mental model is essentially an internal representation or image the structure of which corresponds in crucial respects to that of the object or situation it represents. Thus a mental model is similar to a physical model of the represented situation, somewhat like a chemist's model of a molecule, or an architect's model of a house, and therefore different from a linguistic expression that describes a situation. As a consequence, a mental model can represent only those properties that can be preserved under the condition of similarity. Remember that to avoid this kind of restriction was a central point motivating the principles of SF. Although SF does not preclude similarity altogether, it does not require it (beyond analogy of hierarchical organization). This freedom allows for SF-representations that in some way correspond to mental models. For the sake of illustration, suppose that (22) is a mental model that represents a situation that could variably be described by (23)(a), (b), or (c), among others:

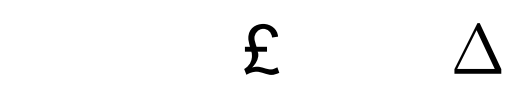

(23) (a) A circle is on the left of a square and a triangle is on the right of the square

(b) A square is on the right of a circle and a triangle on the right of the square

(c) A circle is on the left of a square, which is on the left of a triangle

\section{[ S INST [ $\varepsilon x[$ CIRCLE X ] ] [ LOC [ LEFT [ $\varepsilon$ y [SQUARE y $]]]]]]$ AND [ $S^{\prime}$ INST [ [ $\varepsilon$ z [TRIANGLE $Z$ ]] [ LOC [ RIGHT [ 1 y [ SQUARE $\left.\left.\left.\left.\left.\left.y\right]\right]\right]\right]\right]\right]$}

In (24), a simplified SF of (23a) is given, where the three objects of (23), represented by a circle, a square, and a triangle in (23a), are identified by indefinite descriptions, viz. [ $\varepsilon \times[$ CIRCLE X ] ], [ $\varepsilon y$ [ SQUARE y] ], and [ $\varepsilon z$ [ TRIANGLE $z$ ] ], respectively. The operator for indefinite reference $\varepsilon$, which appears in these descriptions, is an element of type $\langle e,\langle t, e\rangle\rangle$, that combines a variable of type e and a proposition of type $t$ into a configuration of type e. Its interpretation introduces an individual, which corresponds to the variable bound by the operator and meets the conditions specified in the proposition. Likewise, the definite description build up by the l-operator takes up an individual already identified. According to these provisional comments, definite and indefinite descriptions may correspond directly to objects of mental models, though both have absolutely different internal structures, corresponding to different aspects of mental organization. This holds equally for the conditions the objects are subject to, in our example the spatial relation represented as is on the left/right of in (23). The

\footnotetext{
${ }^{21}$ It is not surprising that terminological matters are not very clear in this respect. Besides the distinction between Logical Form and Semantic Form discussed above (both of which must be construed as versions of propositional structure), Jackendoff (1996) uses the term Conceptual Structure CS to capture essentially the same aspect of meaning that I have called SF. Reviewing relevant parts of the literature on visual perception, Jackendoff makes a sharp distinction between CS and Spatial Representation SR. This distinction is very similar to that between SF and spatial representations advocated below, or between propositional representations and mental models.
} 
mental model (22) exhibits these relations not by elements of representation, but directly by the mode of combination, similar to the way in which e.g. /tik/ and /kit/ are different in PF. In SF, however, [ x LOC [ LEFT y ] ] and [ z LOC [RIGHT y ] ] do not only turn these spatial relations into explicit representational elements, but do, moreover, split them up into the common (and completely abstract) condition of localization, represented by the element LOC of type $\langle e,\langle e, t\rangle\rangle$, and the specification LEFT vs. RIGHT of type $\langle$ e.e $\rangle$, which assigns the corresponding region to its argument.

As one easily realizes, the correspondence between SF and mental models is anything but simple and obvious, even in artificially simple cases like (23). It should therefore be emphasized, that the format of these representations is determined by independently motivated crucial conditions: SF has to accommodate, as already noted, the full range of modules participating in $\mathrm{C} / \mathrm{I}$, and must adapt them to the computational system of recursive symbol combination. The principles of mental models on the other hand depend on central aspects of the sensory input, and they directly support certain types of reasoning. Thus, Johnson-Laird (1994) has shown, that for instance decisions about spatial relations between objects are made on the basis of "direct inspection" applied to mental models like (22), rather than by means of logical rules operating on propositional representations deriving from (23). ${ }^{22} \mathrm{~A}$ different aspect of mental models and a more intricate kind of operation than direct inspection is involved in the phenomena called "mental rotation", reported e.g. by Shepard and Cooper (1982). According to these studies, the time to decide about the identity of two geometrical objects, presented by two-dimensional diagrams, depends directly on the size of the angle by which one of these objects is rotated against the other.

The full range of insights and problems related to visual images and mental models goes far beyond the present limits, as is clear e.g. from the concise survey given by Osherson et al. (1990). But whatever aspects might turn out to be relevant for mental representations of visual and related experiences, their interaction with the language dependent Semantic Form is subject to at least two un-escapable limitations.

First, there is a fundamental, inherent boundary for mental models of any kind relative to SF (or other sorts of propositional representation), due to the condition that the structure of mental models must correspond to the structure of what they represent. The most elementary consequence is that mental models cannot represent propositional negation. Thus although (22) and numerous other mental models would be compatible with e.g. (25), there is, by definition, no mental model that actually represents this situation.

(25) There is no circle to the right of the triangle.

\footnotetext{
${ }^{22}$ The type of reasoning explored by Johnson-Laird is illustrated by (i) $-(v)$, where the answer to $(v)$ is based on the mental model in (vi)

(i) The knife is on the right of the plate.

(ii) The spoon is on the left of the plate.

(iii) The fork is in front of the spoon.

(vi) $S \quad P \quad K$

(iv) The cup is in front of the knife.

(v) What's the relation between the fork and the cup?

That in fact models like (vi) are used to answer (v) is shown by descriptions that do not yield an unequivocal model representation, forcing the use of rules for propositional inferences, which causes remarkable delay and errors.
} 
The crucial point is the operator NOT, provided by SF and realized in PF in various, grammatically determined ways. This possibility depends on the fact that natural languages are combinatorial systems of symbols with arbitrary, non-iconic correspondence between SF and PF. It is exactly the iconic, non-arbitrary nature of mental models, which excludes an explicit counterpart of the operator NOT in the representational system of mental models. It is worth noting, moreover, that NOT differs substantially even from SF-elements like LOC or LEFT and RIGHT. Although these elements do - like NOT - lack explicit counterparts in mental models, they still correspond to relations (or regions) within the spatial structure from which the representational format of mental derives, ${ }^{23}$ while NOT cannot correspond to any particular aspect of a mental image. It merely determines its "validity" as a whole, relative to some other representation.

This limitation of mental models infects a large range of conditions and distinctions that can be represented in SF, but lack direct counterparts in mental models. This includes central aspects of modality, time, conditionality, and abstract properties like familiarity, moral value, etc. Consider simple cases like (26):

(26) (a) The car does not move.

(c) The car will move shortly.

(b) The car can move.

(d) The car would move, if necessary

These sentences would all be compatible with, but not represented by the mental image of a car that doesn't move. And the difference between The car is moving and The car was moving is hardly represented by two systematically differing mental models. How far these limitations extend, is difficult to decide. A mental model that explicitly represents the content of The next war will be awful is hard to imagine ${ }^{24}$. In any case, even if mental models could use space as a kind of analogical structure, representing e.g. social hierarchies as configurations in a "social space", there are systematic limits with respect to the representational capacity of mental models.

Second, there is an equally principled limitation of SF with respect to characteristic distinctions and conditions appearing in mental models or visual images. The most striking, but certainly not the only case in point is the domain of faces: Every normal person can distinguish, remember, and identify the faces of an indefinite number of individuals under extremely different circumstances. Many of these individuals may be named and described, but their faces can never be verbalized. Faces can be characterized by diagnostic features and properties, but the actual identification of a face succeeds only by visual means. Similar observations apply, incidentally, to voices, identifying people by auditory means. Cartoons and caricatures furthermore indicate possibilities for genuine, non-propositional abstractions and cognitive operations on mental images representing faces. The point here is not the interesting question whether and to what extent faces are special, as opposed to other domains

\footnotetext{
${ }^{23}$ It must be added, that even elements like SQUARE or TRIANGLE, which incidentally are not true primes, but configurations of SF, do not correspond directly to objects of mental models, but rather to their charateristic properties. The actual correspondence between SF and mental models in the case of objects involves, as already mentioned, the (in)definite descriptions and the referential variables on which they are based. These are important aspects of the different representational principles on which visual images and prepositional structures are based.

${ }^{24}$ The problem is, of course, not the possibility to imagine any sort of horrible scenarios from former or fictitious wars, but the abstract qualification of a potential future war. Without stretching the notion of structural correspondence far beyond the reliable conditions assumed so far, no mental model that properly corresponds to what it represents is available for a statement about abstract states of affairs.
} 
of visual patterns, but that $\mathrm{C} / \mathrm{I}$ represents sensory distinctions that do not correspond to elements or distinctions represented in SF.

An important consequence of these observations concerns the principle of expressibility mentioned earlier. What can be expressed in a given natural language in the sense that the Phonetic Form of an expression corresponds to a distinct representation in SF includes only the propositional aspect of all mental representations. In other words, the "mental world" may well contain thoughts, which can only indirectly be represented by a linguistic expression.

The problems indicated so far would easily multiply, if one thinks of further specialized modules participating in $\mathrm{C} / \mathrm{I}$, such as music, emotions, or interpersonal relations, to mention just three fairly well studied and rather different domains. To enter the details of the respective representations and mental operations would not only go far beyond the present limits; it is actually unfeasible, since explicit proposals, as far, as they are available, are too disparate to allow different principles of representation to be compared and interrelated. For the domain of music, Jackendoff and Lehrdal (2005) have made revealing proposals about the mental representation of the formal structure of musical utterances. It seems to be parallel and in fact related to the prosodic aspect of PF in relevant respects, i.e. to meter and stress of natural language. Whether and how the structures of music represent somehow those of motion and emotion, is an intriguing, but unsettled question. ${ }^{25}$ I must refrain here from even more inconclusive speculations about representational conditions of emotions, Other Minds, and further domains of mental representation.

Two things should be clear, however, even from these highly provisional remarks. First, the different modules of $\mathrm{C} / \mathrm{I}$ must have specific, representational systems with different elements and modes of combination, complying, among others, with their different types of input. They cannot, under this perspective, constitute a single, homogeneous mode of mental organization ${ }^{26}$. And second, in spite of the alleged heterogeneity, the separate sub-systems must interact in specific, systematic, and normally spontaneous ways, independent of linguistic expressions. In other words, there must be appropriate representations in terms of which different modules can cooperate. Visual control of motoric patterns, or the activity of different systems jointly constituting the system of spatial orientation are obvious examples. ${ }^{27}$

The intriguing question emerging from these considerations is: How do different systems of $\mathrm{C} / \mathrm{l}$ interact with SF? Does e.g. the visual system, which must integrate, among others, colors, shapes, positions and motion of objects, interact with SF directly, i.e. in terms visual patterns and images, or does it contribute to a more comprehensive, not domain-specific sort of representation, which eventually provides the interpretation of SF? This leads to at least two alternative possibilities:

\footnotetext{
${ }^{25}$ In Bierwisch (1979), I have suggested that the formal structure of music is iconically related to what might be called "Gestural Form", comparable to the fact that Phonetic is symbolically related to Semantic (or Logical) Form. Gestural Form would thus be in a way the meaning assigned to musical form. In any case, music has a specific relation to emotion. It is not about emotion, but represents it. ${ }^{26}$ There is, of course, after all we know a single and uniform manner of representation in terms of neurons, their synaptic connection, and the patterns of activation they allow for. But that is not the relevant level of analysis in the present context.

${ }^{27}$ A parallel case in point is the integration of articulation and auditory perception constituting the interface-partner for PF, as noted earlier.
} 
(27) Assuming, that human behavior is based on a complex array of autonomous systems of mental organization with domain specific types of representation requires that either

(a) SF serves as the interface in terms of which the different, otherwise separate systems of $\mathrm{C} / \mathrm{l}$ interact, or

(b) $\mathrm{C} / \mathrm{I}$ provides different types of interaction for its systems, hence SF draws on (at least partially) integrated $\mathrm{C} / \mathrm{I}$-representations.

Although these options seem to define a rational alternative - SF either creates or is imposed on the interaction of different modules -, it is difficult to see, how it could be decided on empirical grounds ${ }^{28}$. However these problems will be solved or perhaps reformulated, an automatic correspondence between SF and the representations in $\mathrm{C} / \mathrm{I}$ is indispensable, it is in fact the essence of the interface-function. The correspondence must obviously satisfy different requirements for the mapping from SF into C/I and from C/I into SF. In what follows, I will nevertheless keep to one perspective as far as possible.

As already noted, the combinatorial structure of SF is open to any interpretation that meets the conditions imposed by the type-based hierarchy. For the sake of illustration, look at the configuration [ LOC [ LEFT [ $\varepsilon$ y [SQUARE y ] ] ] in (24), where the interpretation of the operator $\varepsilon y$ introduces an instance of $y$, i.e. an individual of type e, which combines with the condition SQUARE identifying a property of this individual. Next the functor LEFT combines with this individual, assigning to it the (spatial) region on its left, which again functions as an entity of type e. Now the element LOC of type $\langle e,\langle e, t\rangle\rangle$ combines with this individual and creates the property of being located within this region. In (24), this property is then assigned to the individual $x$ which has the property CIRCLE. As noted above, one way to think of the interpretation corresponding to (24) is the mental model indicated in (22), which shows that elements of SF get interpretations with rather different status in $\mathrm{C} / \mathrm{I}$. Thus, while the combinatorial structure of SF is transparent in the sense that it does not fix anything beyond connectedness, the "content" of this connection as well as any other condition entering the conceptual interpretation is determined by the basic elements of SF. Hence any condition an SF-representation imposes on its interpretation within the framework of type structure is ultimately due to the basic elements of SF.

\footnotetext{
${ }^{28}$ A decided position is advocated by Fodor (1983) on theoretical grounds. He conceives of the human mind as consisting of autonomous, domain-specific input/output-systems, which support a central system, which is global, fully interactive and mediates between the otherwise encapsulated inputmodules. On Fodor's view, language is one of the autonomous input-output-modules, which do not interact with other modules, but only with the central system. In spite of its clear and definite position, it is unclear, however, how SF (or LF, for that matter) is to be conceived under this view: Either SF is assumed to participate in mediating among the various subsystems of the mind, analogous to (27a). This would mean, however, that it must belong to the central system and could by definition not be part of language, which is one of the autonomous input-output modules. Or SF belongs to language, as independent reasons clearly suggest, but then it cannot subserve the interaction with other modules, similar to (27b). In order avoid these and other problems, Jackendoff (1997) proposed, what he calls "Representational Modularity". On this view, modules are not encapsulated input- or outputsystems, like language comprehension or visual perception. Modules are rather construed as autonomous representational systems. Thus PF or the Syntactic Structure SS are modules, alongside with e.g. visual or musical representation. How SF (or the Conceptual Structure CS, which corresponds to it in Jackendoff's system) as a module relates to other representations in C/l is not obvious, though. The view on Spatial Representation and its relation to CS presented in Jackendoff (1996) seems to favor the position of (27b). The issue requires further discussion, though.
} 


\section{Three Kinds of Basic Elements of SF}

The nature of these basic semantic elements is among the most controversial issues in semantic theory. There is little reason to assume that it can be settled just by inspecting the interface properties of semantic representations. It might be useful, though, to sort out some distinctions with respect to the way basic elements participate in the organization of SF-representations and their interpretation in $\mathrm{C} / \mathrm{l}$.

Although lexical items are the building blocks from which complex linguistic expressions are made up, their Semantic Form, as noted above, is not in general a single basic element, but a more or less complex configuration of more elementary units. One kind of these elementary units are elements that have repeatedly been identified because of their fairly obvious syntactic or semantic effects. Thus characteristic syntactic and semantic properties of causative verbs such as kill, give, show, change, close and many others are captured by elements like ACT and CAUSE, as indicated in (21a), and extensively discussed e.g. in Wunderlich (1997). Likewise, characteristic semantic (and syntactic) properties of color terms like red, blue, etc.

are determined by the element COLOR, as shown in (21b). ${ }^{29}$ Kinship terms like father, sister, son, parent are another domain the syntactic and semantic properties of which are determined by systematic combinations of primes like MALE, PARENT, ANIMATE. Further areas of lexical structure, like terms for spatial dimensions, (change of) possession, would easily provide additional evidence for an array of systematic primes. The morpho-syntactic effects of elements of this sort include, besides Argument Structure with categorial and semantic selection, conditions on Person, Number, Gender, Tense, Mood, and others. A provisional cut-out of the system of these elements is given in (28), where elements are grouped according to their typemembership.

(28) Systematic Primes
(a) HUMAN, MALE, ALIVE, OPEN, ACT, ....
$\langle e . t\rangle$
(b) LOC, COLOR, PARENT, SEE, HAVE, ....
$\langle e,\langle e, t\rangle\rangle$
(c) CAUSE, AND, ....
$\langle\mathrm{t},\langle\mathrm{t}, \mathrm{t}\rangle\rangle$
(d) NOT, BECOME, POSSIBLE, ....
$\langle t, t\rangle$
(e) LEFT, RIGHT, MAXIMAL, VERTICAL ....
$\langle e, e\rangle$

The combinatorial options within SF follow from the type these elements belong to. Their interpretation in $\mathrm{C} / \mathrm{l}$ however depends on varying conditions determined by the modules of $\mathrm{C} / \mathrm{l}$. Some of these conditions were mentioned with respect to mental models as one of the candidates for $\mathrm{C} / \mathrm{l}$-interpretation. There are, of course, lots of intriguing questions to be answered with respect of the conditions fixed by HUMAN, MALE, HAVE, OPEN, etc. What is the core of interpretation, what is the range of variation, how is it determined in different combinations or contexts? Besides these questions, which need to be answered for each of the elements in particular, there are problems, which concern the system as a whole. What is the basis of the

\footnotetext{
${ }^{29}$ Notice that color terms differ from other adjectives also with respect to their syntactic behavior. Thus while for (i)(a) there is the parallel (i)(b), no parallel of this sort exists for (ii)(a):

(i)(a) the blue book, the red shirt

(b) the blue of the book, the red of the shirt

(ii)(a) the interesting book, the long shirt

(b) *the interesting of the book, the long of the shirt

There is, of course, the length of the shirt, but that is a nominalization, which clearly differs from (ib).
} 
elements, could there be arbitrary amendments, or is the system closed and fixed, how is it determined ontogenetically, i.e. what input is needed to activate its elements? To assume a systematic, perhaps universal status for the overall system, of which (28) is an incidental illustration, does neither imply nor exclude, however, that these elements are part of the biologically fixed language faculty. It might well be the case, that the language faculty determines the dimensions or coordinates along which - within the combinatorial type system - basic elements of SF can be differentiated, without providing a repertoire that is fixed a priori.

The items illustrated in (28) are systematic basic elements, because of their characteristic role within SF and in I-Language in general. Several of these elements have been analyzed under various perspectives in the literature. The precise delimitation of this system is, of course, not obvious at all. The core of it is fairly clear, however.

Whatever the correct choice of the systematic elements for a given language might be, it is equally clear that the full range of lexical items can hardly be characterized in terms of these elements alone, if the system (28) belongs to is not open for just arbitrary expansion. As mentioned earlier, idiosyncratic distinctions are indispensable, in order to keep apart lexical items like dog vs. cat, horse, tiger, or lion, all of which are based on the systematic element ANIMAL. Likewise red must be distinguished from pink and brown within the set of terms specified by the systematic element COLOR. Since this kind of specificity holds for large parts of the lexicon, recourse to analyses in terms of systematic elements has been rejected as circular e.g. by Fodor (1981), because the system of basic elements would approach the size of the set of lexical items. Hence decomposition of lexical items in terms of basic elements would be circular and should hence be discarded in favor lexical items without lexical decomposition. However, the fact that idiosyncratic elements might be necessary for many, or possibly most lexical items does by no means preclude them from exhibiting compositional structures based on idiosyncratic as well as systematic elements, where the letter do in fact provide the frame for idiosyncratic distinctions. The case of color terms illustrated by $(21 \mathrm{~b})$ is one of the more obvious examples ${ }^{30}$.

Idiosyncratic elements, introduced for the first time as "distinguishers" in Katz and Fodor (1963), reappeared in a somewhat different guise in Pustejovsky (1995) under the slightly misleading title "Qualia structure". As a matter of fact, two fairly different kinds of idiosyncratic elements must presumably be distinguished according to a number of criteria, among them the different background or origin in $\mathrm{C} / \mathrm{l}$. As a rough orientation, idiosyncratic elements might either be irreducible, i.e. routed in primary sensory qualities, or inducible, i.e. constructed on the basis of specific and cumulative information. I will call them Qualifying elements and Dossiers, respectively, and briefly illustrate them in turn.

To begin with, Qualifying elements are meant to specify for example values within perceptual domains corresponding to lexical items like sweet, sour, bitter, cold, hot, hard, soft, dark, bright, red, blue, etc. The perceptual domains as such might be identified by Systematic elements, which set the stage for idiosyncratic distinctions

\footnotetext{
${ }^{30}$ Semantic elements representing different color shades are not only classified by means of the systematic, relational component COLOR, they are furthermore subject to systematic constraints governing their possible verbalization. As shown by Berlin and Kay (1969), conceptualization and verbalization are strongly dependent on cardinal colors structuring the perceptual field of color.
} 
marked by be Qualifying elements, as hinted at for color terms. There is a wide range of intriguing problems concerning the origin, the status, and the effect of the perceptual domains and distinctions, most of which, though clearly part of $\mathrm{C} / \mathrm{l}$, are outside the range of language and SF. On the other hand, problems of metaphorical use of lexical items relying on synesthetic conditions, if e.g. dark and bright shift from sensory to intellectual, ethical or mood qualities, may well be a particular issue of interface effects.

A further intricate issue is the status of complex perceptual patterns such as the voice or the face of a person or the shape of a particular objects like leaves, flowers, or animals. As noted earlier, sensory clusters of this sort are available for cognitive operations - they can be remembered, recognized, modified, labeled -, but their content can generally not be verbalized, i.e. it cannot be represented by a compositional verbal expression. This raises the question whether clusters of this sort are to be considered as Qualifying elements, in case they are connected to a representation in I-Language. If so, the representation of a familiar person in SF includes a Qualifying element, whose interpretation in $\mathrm{C} / \mathrm{l}$ is the visual pattern representing the face (and the voice and perhaps characteristic gestures etc.) of that person. These considerations lead directly to the intriguing question, how visual (and other sensory) patterns serving as prototypes for conceptual classification are to be dealt with. This is, of course, a far-reaching problem that cannot seriously be taken up here.

Turning next to what I have called Dossiers, i.e. basic elements constructed and enriched according to experience. An obvious way to illustrate the nature of these elements is to look at proper names, which have an SF, that must characteristically include an element of the kind in question. Starting with the assumption that Berlin means something like the city named "Berlin", ${ }^{31}$ one notes that the SF of a name must comprise four characteristic components, as indicated in (29), where (d) consists of the conditions that make up the Dossier about Berlin, its geographical position, its function, etc., which is not only necessary to distinguish the various places with the same name, but also to fix the properties the speaker does rely on in identifying the place.

(29) The SF of a normal Proper Name specifies

(a) Referential definiteness, i.e. representation of a fixed individual $x$

(b) Classification of $x$ as an instance of a certain class (like PERSON, PLACE, etc.)

(c) Assignment of the name as a property of $x$

(d) Assignment of whatever is (assumed to be) known about $x$

To illustrate this schema, the lexical entry for Berlin would look like (30), where ix identifies a specific individual within the representational space of $\mathrm{C} / \mathrm{I}$, which is classified as a place, for which the Phonetic Form is made accessible by PF to the naming component, i.e. the SF-condition, by which a proper name is assigned to $x^{32}$ and BERLIN is a set of conditions which jointly characterize $x$ :

\footnotetext{
${ }^{31}$ There is a vast literature about the diverse problems of proper names, which need not be taken up here. For some discussion of the relevant aspects see e.g. Geurts (1997).

${ }^{32}$ This is a provisional convention, which must eventually account for potential PF-variants of a name, and also for diagnostic conditions, to correlate and distinguish graphemic variants or alternatives. But these matters can be ignored here.
} 
(30) /berli:n/ [+N, -Masc, -Plur...]

$[\mathrm{lX}[[$ PLACE $X]$ AND [PF NAME-OF X ] AND [BERLIN X ] ]]

Three points are to be noted with respect to BERLIN and Dossiers in general, including in particular those about persons. First, a Dossier might contain all types of information that are available in $\mathrm{C} / \mathrm{I}$ and permanently associated with the individual $\mathrm{x}$. The Dossier might in particular include visual or other sensory representations bound to $x$, but just as well abstract or propositional conditions, like age, or time and manner of acquaintance. Second, a Dossier in SF is a prime, which means that it functions as a single, unanalyzed, encapsulated element, just like Systematic or Qualifying elements. But it can, so to speak, become transparent to the extent to which the content of the Dossier can be matched by the representational format of SF. That is possible for propositional conditions, but barred for the internal structure of perceptual patterns. Hence a Dossier may be a kind of sluicing device which allows information from C/I to appear in SF. In other words, Dossiers are primes like other basic elements, their internal make up is closed off, but it is available for representation in SF as far as it meets the representational format of SF. And third, it follows from the internal structure of Dossiers, that the system of these elements can be expanded not only with respect to each individual Dossier as a specific element by new information to be incorporated - but also with respect to the whole repertoire, by introducing new elements, which characterize additional individuals.

Now, the next step is to observe that proper names are not special in using dossiers. Idiosyncratic conditions CANINE, FELINE, or EQUINE, distinguishing dog, cat, and horse on the basis of the systematic element ANIMAL, are in all respects just noted Dossiers like those of BERLIN, PARIS, NAPOLEON, or HANS CASTORP. They would include, in particular, the visual pattern of a prototypical cat, dog, or horse, and they would indicate conditions on typical behavior and species specific sounds. In a similar vein, the Dossier of book or knife collects conditions on shape and functional aspects. A great deal of the revealing analyses in terms of qualia structure, co-composition, and coercion in Pustejovsky (1995) are based on conditions of this sort. Thus, in order to interpret Mary finished the book, the qualia structure must provide the condition that a book can be written and read, and that both activities can begin and have an end, so Pustejovsky argues, which supports the (at least) twofold conceptual interpretation of the sentence.

Let me finally mention two non-trivial ways in which Dossiers and Qualifying elements are interrelated. First, as just noted, visual and other sensory representations - faces, prototypical shapes, voices, characteristic calls - and hence potential Qualifying elements may naturally be included as proper parts into Dossiers. Second, Qualifying elements, initially construed as distinctions within basic domains of sensory representations and hence as simple perceptual values directly determined by receptors or effectors, can obviously acquire fairly complex structure, resulting from higher order integration of sensory input or motoric control. Thus faces and complex shapes are anything but simple values of sensory domains, they rather result from exposition to complex information under varying conditions. Hence the borderline between Qualifying elements and (certain aspects of) Dossiers might not be as sharp as their essentially different nature would suggest. And Pustejovsky might not be too far off the mark in lumping them together under a single heading. 


\section{Instead of Conclusions}

It is easy to see that there are unanswered questions, even with respect to the central issues of the present considerations. It nevertheless seems to me fairly safe to assume that semantic representations are based on an abstract, transparent, combinatorial structure. As a matter of fact, all reasonable approaches to semantics are based on this assumption in one way or the other. It is also obvious that the representations making use of a framework of this kind must interact with different mental subsystems in terms of which the human mind organizes its experiences and actions. The remaining question concerns the origin and nature of the packaging, so to speak, of the units that mediate between SF (or its equivalent in other approaches) and its external interpretation. The conjecture defended here is the assumption that the elements realizing this interface business are of roughly three sorts, rooted either in the computational system of language (systematic elements), or in the external, primarily sensory systems (Qualifying elements), or participating in both, as a kind of sluicing mechanism (Dossiers).

The assumption of Dossiers, living on some sort of double currency, is less illegal as it might appear at first glance. If one recognizes the fact, that the language faculty and the representations it supports, is phylogenetically as well as ontogenetically imposed on other, independently operating mental systems, recruiting them as domains of interpretation, then a compromise is natural under certain conditions, and it is likely to take a form like the one envisaged here. The need to spell out the details and consequences leaves us with a lot of open questions.

\section{References}

Berlin, Brent, and Paul Kay (1969) Basic Color Terms, Berkeley and Los Angeles: University of California Press

Bierwisch, Manfred (1979) Musik und Sprache, in: Jahrbuch Peters 1978, S. 9-102, Leipzig: Edition Peters

Bierwisch, Manfred (1997) Lexical Information from a Minimalist Point of View, in: Christopher Wilder, Hans-Martin Gärtner, Manfred Bierwisch (eds.) The Role of Economy Principles in Linguistic Theory, 227-266, Berlin: Akademie-Verlag

Bierwisch, Manfred (2001) Repertoires of Primitive Elements - Prerequisite or Result of Acquisition? in: Weissenborn and Höhle (eds.) Approaches to Bootstrapping, Vol 2. p. 281-307, Amsterdam: John Benjamins

Bierwisch, Manfred and Rob Schreuder (1992) From concepts to lexical items, in: Cognition 42, 23-60.

Bloom, Paul, Mary Peterson, Lynn Nadel, and Merrill Garrett (eds.) (1996) Language and Space, Cambridge, Mass.: MIT Press 
Bowerman, Melissa (1996) Learning How to Structure Space for Language: A Crosslinguistic Perspective, in: Paul Bloom et al.(eds.) Language and Space, 385-436

Chomsky, Noam (1995) The Minimalist Program, Cambridge, Mass.: MIT Press

Dowty, David R. (1979) Word Meaning and Montague Grammar, Dordrecht, Holland: D. Reidel

Fodor, Jerry A. (1981) Representations, Cambridge, Mass.: MIT Press

Fodor, Jerry A. (1983) The Modularity of Mind, Cambridge, Mass.: MIT Press

Frege, Gottlob (1879) Begriffsschrift, eine der arithmetischen nachgebildete Formelsprache des reinen Denkens, Halle a.S.: L. Nebert

Geurts, Bart (1997) Good News about the Description Theory of Names, Journal of Semantics, 14, 319-348

Hale, Kenneth and Samuel Jay Keyser (1993) On Argument Structure and the Lexical Expression of Syntactic Relations, in: Kenneth Hale and Jay Keyser (eds) The View from Building 20, 53-109, Cambridge, Mass.: MIT-Press

Halle, Morris (1983) On Distinctive Features and their Articulatory Implementation, Natural Language and Linguistic Theory, 1, 91-105

Halle, Morris (1995) Feature Geometry and Feature Spreading, Linguistic Inquiry 26, $1-46$

Jackendoff, Ray S. (1996) The Architecture of the Linguistic-Spatial Interface, in: Paul Bloom et al.(eds.) Language and Space, 1-30

Jackendoff, Ray S. (1997) The Architecture of the Language Faculty, Cambridge, Mass.: MIT Press

Jackendoff, Ray S. and Fred Lehrdal (2005) The Capacity for Music:What Is It and What's Special About It? to appear in: Cognition

Johnson-Laird, Philip N. (1996) Space to Think, in: Paul Bloom et al.(eds.) Language and Space, 437-462

Katz, Jerrold J (1972) Semantic Theory, New York: Harper and Row

Katz, Jerrold J. and Jerry A. Fodor (1963) The Structure of a Semantic Theory, Language, 39, 170-210

Levinson, Stephen C. (1996) Frames of Reference and Molyneux's Question: Crosslinguistic Evidence, in: Paul Bloom et al.(eds.) Language and Space 109-169 
Montague, Richard (1974) Formal Philosophy, Selected Papers, edited by Richmond H. Thomason, New Haven: Yale University Press

Osherson, Daniel N., Stephen M Kosslyn, John M. Hollerbach (eds.) (1990) Visual Cognition and Action, Cambridge, Mass.: MIT Press

Partee, Barbara H, Alice ter Meulen, Robert E. Wall (1990) Mathematical Methods in Linguistics, Dordrecht: Kluwer Academic Publishers

Pustejovsky, James (1995) The Generative Lexicon, Cambridge, Mass.: MIT Press

Schnelle, Helmut (1999) Mental Computation - A Critical Analysis of some Proposals by M. Bierwisch, Theoretical Linguistics, 25, 257-281

Shepard, Roger N. and Lynn A. Cooper (1982) Mental Images and their Transformations, Cambridge, Mass.: MIT Press

Searle, John R. (1969) Speech Acts, Cambridge: Cambridge University Press

Wunderlich, Dieter (1997a) Cause and the structure of verbs, Linguistic Inquiry, 28, 27-68

Oxford Advanced Learner's Dictionary of Current English, 11. edition (1980), London: Oxford University Press

The American Heritage Dictionary of the English Language (1992), Boston: Houghton Mifflin Co. 